\title{
Increased frequency of CD16+ monocytes and the presence of activated dendritic cells in salivary glands in primary Sjögren syndrome
}

\author{
M E Wildenberg, ${ }^{1} \mathrm{~J}$ M C Welzen-Coppens, ${ }^{1}$ C G van Helden-Meeuwsen, ${ }^{1} \mathrm{H}$ Bootsma, ${ }^{2}$ \\ A Vissink, ${ }^{3} \mathrm{~N}$ van Rooijen, ${ }^{4} \mathrm{~J}$ P van de Merwe, ${ }^{1} \mathrm{H}$ A Drexhage, ${ }^{1} \mathrm{M}$ A Versnel ${ }^{1}$
}

\begin{abstract}
- Supplementary table 1 is published online only at http:// ard.bmj.com/content/vol68/ issue3

${ }^{1}$ Department of Immunology, Erasmus MC, Rotterdam, The Netherlands; ${ }^{2}$ Department of Rheumatology and Clinical Immunology, University Medical Center Groningen, Groningen, The Netherlands; ${ }^{3}$ Department of Oral and Maxillofacial Surgery, University Medical Center Groningen, Groningen, The Netherlands; ${ }^{4}$ Department of Molecular Cell Biology, Vrije Universiteit, Amsterdam, The Netherlands
\end{abstract}

Correspondence to:

M E Wildenberg, Department of Immunology, Erasmus MC, P.O.

Box 2040, 3000 CA Rotterdam, The Netherlands;

m.wildenberg@erasmusmc.nl

Accepted 30 March 2008 Published Online First 8 April 2008

\section{ABSTRACT}

Objectives: In the salivary glands of patients with primary Sjögren Syndrome (pSjS) an accumulation of dendritic cells (DCs) is seen, which is thought to play a role in stimulating local inflammation. Aberrancies in subsets of monocytes, generally considered the blood precursors for DCs, may play a role in this accumulation of DCs. This study is aimed at determining the level of mature CD14 ${ }^{\text {low }} \mathrm{CD} 16+$ monocytes in pSjS and their contribution to the accumulation of DCs in pSjS.

Methods: Levels of mature and immature monocytes in patients with pSjS $(n=19)$ and controls $(n=15)$ were analysed by flow cytometry. The reverse transmigration system was used for generation of DCs generated from monocyte subsets. The phenotype of DCs in pSjS salivary glands was analysed using immunohistochemistry. In vivo tracking of monocyte subsets was performed in a mouse model.

Results: Increased levels of mature CD14 ${ }^{\text {low }} \mathrm{CD} 16+$ monocytes were found in patients with pSjS (mean (SD) 14.5 (5.5)\% vs 11.4 (3.4)\%). These cells showed normal expression of chemokine receptor and adhesion molecules. Mature monocytes partly developed into DClysosome-associated membrane glycoprotein (LAMP)+ (19.6 (7.5)\%) and CD83+ (16 (9)\%) DCs, markers also expressed by DCs in pSjS salivary glands. Monocyte tracking in the non-obese diabetic (NOD) mouse showed that the homologue population of mature mouse monocytes migrated to the salivary glands, and preferentially developed into CD11c+ DCs in vivo.

Conclusions: Mature monocytes are increased in pSjS and patient and mouse data support a model where this mature monocyte subset migrates to the salivary glands and develops into DCs.

Primary Sjögren syndrome $(\mathrm{pSjS})$ is an autoimmune disorder characterised by focal lymphocytic infiltrates in the salivary and lachrymal glands. ${ }^{1}$ Although the pathogenesis remains to be fully elucidated, several findings indicate involvement of the innate immune system. These include increased expression of Toll-like receptors and upregulation of the type I interferon (IFN) system. ${ }^{2-5}$ Furthermore, in labial biopsies, increased numbers of dendritic cells (DCs) were observed. ${ }^{6}$ Interestingly, in the non-obese diabetic (NOD) animal model for $\mathrm{pSjS}$, a strong accumulation of DCs was observed prior to the appearance of infiltrates. ${ }^{7}$ DCs are potent antigen-presenting cells and play a key role in the development of immune responses by stimulating $\mathrm{T}$ cells and boosting $\mathrm{B}$ cell responses. ${ }^{8-10}$ Therefore, the increased presence of
DCs is likely to play a role in the initiation and perpetuation of local immunity.

The increased presence of DCs in the $\mathrm{pSjS}$ salivary gland may involve aberrancies in the precursor population of blood monocytes. Interestingly, monocyte alterations have been described in other autoimmune conditions such as rheumatoid arthritis (RA) and diabetes, and include increased expression of Fc receptors, increased adhesion to extracellular matrix and shifts in monocyte subsets. ${ }^{11-13}$ In humans, monocytes can be separated in two populations, based on expression of CD14 and CD16. The majority of monocytes are CD14+CD16-, but approximately $10 \%$ are $\mathrm{CD} 14^{\text {low }} \mathrm{CD} 16+$ and this is considered to be a relatively mature population. ${ }^{14}{ }^{15} \mathrm{CD} 16+$ mature monocytes are further characterised by high levels of CX3CR1 and low levels of chemokine (C-C motif) receptor 2 (CCR2) and CD62L. ${ }^{16} \mathrm{CD} 16-$ immature monocytes have been hypothesised to convert into mature $\mathrm{CD} 16+{ }^{17}$ which in turn develop into DCs. Indeed, CD16+ monocytes have been shown to preferentially develop into tissuetrafficking DCs in an in vitro model. ${ }^{17}$ In the mouse system, the populations of Ly- $6 \mathrm{C}^{\text {high }}$ and Ly- $6 \mathrm{C}^{\text {low }}$ monocytes are considered to be homologous to the human CD16- and CD16+ subsets. ${ }^{15}$ Supporting the idea of a kinetic relationship between the two subsets, in mice it has been shown that in vivo, Ly$6 C^{\text {high }}$ monocytes develop into Ly-6Clow monocytes. ${ }^{18}$

Previously, increased levels of mature monocytes were described in RA and infectious diseases such as HIV and sepsis. ${ }^{13}{ }^{19-21}$ In human pSjS, no data has been reported on the levels of mature monocytes. However, in the NOD mouse, ${ }^{22}$ the mature monocyte subset is increased significantly. ${ }^{23}$ The purpose of this study was to determine if the mature monocyte subset is expanded in $\mathrm{pSjS}$ and if DCs derived from this subset contribute to the accumulation of activated DCs observed in the salivary glands of these patients.

\section{MATERIALS AND METHODS}

\section{Patients and controls}

Heparinised blood samples were obtained from 19 female patients with $\mathrm{pSjS}$ fulfilling the AmericanEuropean consensus criteria ${ }^{24}$ (mean (SD) age 52.5 (16.8) years, mean disease duration 8.2 (9.1) years) at Erasmus MC, Rotterdam, The Netherlands (see Supplementary material). Patients treated with corticosteroids were excluded. Disease duration was determined as time from initial diagnosis to 
time of inclusion. Control samples were obtained from 15 female volunteers (mean age 46.2 (6.0) years) not suffering from autoimmune diseases or taking immunosuppressive medication. Labial biopsies of six subjects were obtained during diagnostic biopsy procedures at the Erasmus MC. Two patients were diagnosed as pSjS according to the criteria mentioned above, and four did not meet any of the objective criteria for $\mathrm{pSjS}$ and were considered controls. Parotid biopsies of two patients fulfilling the criteria for $\mathrm{pSjS}$ were obtained at the University Medical Center Groningen, Groningen, The Netherlands in the course of another study. ${ }^{25}$ All subjects gave informed consent and experimental procedures were approved by the Medical Ethical Committees of Erasmus MC and University Medical Center Groningen.

\section{Mice}

NOD/LTj mice were bred and housed in our own facility under specified pathogen-free conditions. All experimental procedures were approved by the Erasmus University Animal Ethical Committee.

\section{Cell isolations}

Peripheral blood mononuclear cells (PBMCs) were isolated from peripheral blood using a Ficoll gradient (Amersham, Little Chalfont, UK). When appropiate, CD16+ and CD16- populations were separated using CD16-microbeads and AutoMACS separation (Miltenyi, Bergisch Gladbach, Germany).

\section{Flow cytometry}

Human cell samples were stained using fluorescein isothiocyanate (FITC) anti-CX3CR1 (MBL International, Woburn, Massachusetts, USA), PE anti-CCR2, PE anti-DC-specific intercellular adhesion molecule-3-grabbing non-integrin (SIGN) (R\&D Systems Inc., Minneapolis, Minnesota, USA), $\mathrm{PE}$ anti-human leukocyte antigen (HLA)-DR, PE anti-CD62L, PE anti-CD80, PE anti-CD86, PE anti-CD16 and APC anti-CD14 (BD Biosciences, San Jose, California, USA), PE anti-CD83 and PE-DC-lysosome-associated membrane glycoprotein (LAMP) (Immunotech, Marseille, France). Murine samples were stained using biotinylated anti-CD11c, followed by allophycocyanin (APC)-streptavidin (BD Biosciences).

\section{Reverse transmigration assay}

Development of DCs from monocytes was studied in a reverse transmigration assay. ${ }^{17}$ Briefly, human umbilical vein endothelial cells (HUVECs) were grown to confluency on a collagen matrix (Allergan, Irvine, California, USA). PBMCs were depleted of naturak killer (NK) cells using CD56-microbeads (Miltenyi). CD16+ cells were then isolated using CD16microbeads and labelled with 5(6)-carboxyfluorescein diacetate $N$-succinimidyl ester (Sigma Aldrich, Deisenhofen, Germany). CD16+ and CD16- cells were remixed and applied to the endothelial layer for $2 \mathrm{~h}$. Afterwards, non-adherent cells were washed away and culture was continued for $48 \mathrm{~h}$. Reverse transmigrated cells were then removed using $1 \mathrm{mM}$ ethylenediaminetetraacetic acid (EDTA). All cultures used Ham F12 culture medium (BioWhittaker, Verviers, Belgium), supplemented with $20 \%$ fetal calf serum (FCS) (BioWhittaker), $50 \mu \mathrm{g} / \mathrm{ml}$ endothelial cell growth supplement (R\&D Systems) and $100 \mu \mathrm{g} /$ $\mathrm{ml}$ heparin (Sigma).

\section{Cytokine measurements}

Levels of interleukin (IL)10, IL1 $\beta$, tumour necrosis factor (TNF) $\alpha$ and IFN $\gamma$ were determined using a human cytokine 25-plex AB Bead kit (Biosource, Camarillo, California, USA). Measurements were performed on a Luminex XYP (Luminex, Austin, Texas, USA) and analysed using StarStation software V.2.0 (Applied Cytometry Systems, Sheffield, UK).

\section{Immunohistochemistry}

Labial and parotid biopsies were snap-frozen in Tissue-Tek (Sakura, Zoetermeer, The Netherlands). Cryostat sections $(6 \mu \mathrm{m})$ were prepared and fixed with cold methanol and acetone. To limit background staining, an avidin/biotin block was used (Vector Laboratories, Burlingame, California, USA). Slides were incubated with anti-DC-LAMP (Immunotech, Marseille, France), anti-DC-SIGN or IgG control (BD Biosciences) followed by biotinylated rabbit anti-mouse IgG and subsequent streptavidin-ABComplex-AP (Dako A/S, Glostrup, Denmark). Alkaline phosphatase (AP) label was developed using new fuchsine staining (Chroma, Stuttgart, Germany). Slides were counterstained with haematoxylin (Merck, Whitehouse Station, New Jersey, USA).

\section{In vivo monocyte tracking}

Monocyte subsets were labelled as described previously. ${ }^{26}$ Briefly, for labelling of Ly-6C $\mathrm{C}^{\text {low }}$ monocytes, mice were injected with Fluoresbrite plain YG 0.5 micron microspheres (Polysciences Inc. Warrington, Pennsylvania, USA) intravenously in the tail vein. Before labelling of Ly-6Chigh monocytes, mice were injected with $\mathrm{Cl}_{2} \mathrm{MDP}$ (dichloromethyl diphosphonate; clodronate) liposomes intravenously in the tail vein. Clodronate was a gift of Roche Diagnostics GmbH, Mannheim, Germany. It was encapsulated in liposomes as described previously. ${ }^{27}$ This results in a transient depletion of blood monocytes, followed by repopulation of Ly-6Chigh monocytes. ${ }^{18}$ At $20 \mathrm{~h}$ later, these mice also received Fluoresbrite microspheres intravenously, thus labelling the Ly- $6 \mathrm{C}^{\text {high }}$ population. At 4 days later, submandibular glands (SMG) were removed and cleared of all adipose tissue and lymph nodes. Tissue was then digested using Liberase (Roche, Woerden, The Netherlands) treatment and cells were washed twice in phosphate-buffered saline (PBS) containing 10\% FCS. Cell suspensions were then analysed by flow cytometry.

\section{Statistics}

For comparison of unpaired data, the Mann-Whitney $U$ test was used. For comparison of paired data, the Wilcoxon signed ranks test was used. Correlations were calculated using Spearman rank test. All analyses were carried out using SPSS software (SPSS, Chicago, Illinois, USA), results were considered statistically significant if $\mathrm{p}<0.05$.

\section{RESULTS}

\section{Increase in mature monocytes in pSjS}

PBMC were stained for CD14 and CD16 and frequencies of mature and immature monocyte subsets were determined by flow cytometry. The frequency of CD16+ mature monocytes was significantly increased in $\mathrm{pSj}$ S compared to healthy controls (fig 1A), while the frequency of CD16-immature monocytes was not altered significantly (data not shown). Several cytokines have been reported to induce the upregulation of CD16 on monocytes in vitro, including IL10. ${ }^{28}$ Although levels of IL10 were increased in the serum of patients with $\mathrm{pSjS}$ 

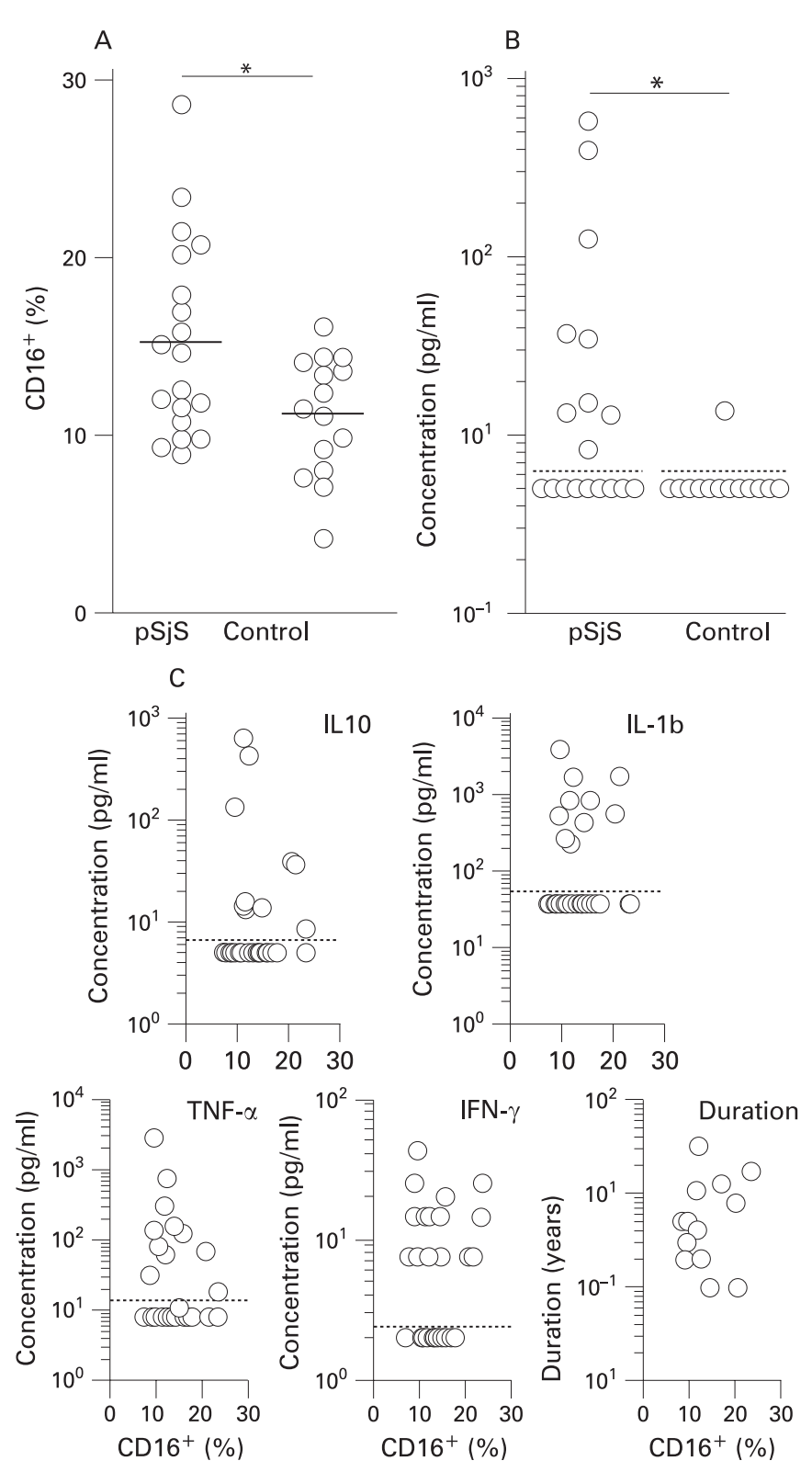

Figure 1 Increased frequency of mature monocytes in primary Sjögren syndrome (pSjS) does not correlate to cytokine levels. A. Peripheral blood mononuclear cells (PBMCs) were isolated, stained for CD14 and CD16 and analysed by flow cytometry. Each data point represents one individual patient, ${ }^{*} p<0.05$. B,C. Serum cytokine levels were measured by 25-plex $A B$ Bead kit. Disease duration was measured as time from diagnosis to inclusion in the study. Each data point represents one individual patient, dashed lines represent detection limits.

(fig 1B), no correlation was found between the level of IL10 and the frequency of CD16+ monocytes (fig 1C, Spearman rho $-0.385, p=0.19$ ). Additionally, levels of the inflammatory cytokines IL1 $\beta$, TNF $\alpha$ and IFN $\gamma$ did not correlate to CD16+ frequency (fig 1C, Spearman rho 0.067, -0.061 and -0.086 , $p=0.72,0.74$ and 0.65 respectively). Furthermore, no correlation between frequency of mature monocytes and disease duration was found (fig 1C, Spearman Rho 0.094, $p=0.76$ ).

\section{Normal phenotype of mature monocytes in pSjS}

Besides expression of CD16, mature monocytes are characterised as CCR2 ${ }^{\text {low }}$ CD62 $L^{\text {low }}$ CX3CR $1^{\text {high }}$. Expression of these markers was determined, as an aberrant expression pattern would have implications for the migration of this subset. Mature monocytes expressed low levels of CCR2 and CD62L and high levels of CX3CR1 (fig 2A). For comparison, immature monocytes expressed low levels of CX3CR1 and high levels of CCR2 and CD62L (fig 2B). No differences were observed between $\mathrm{pSjS}$ and controls, indicating that the monocyte subsets are phenotypically normal in $\mathrm{pSjS}$ with regard to these migration markers and would be expected to show a normal migration pattern.

\section{Mature monocytes are capable of developing into DC-LAMP+ dendritic cells}

The mature monocyte subset has been described to preferentially develop into tissue trafficking $\mathrm{DCs},{ }^{17}$ but the exact phenotype of these DCs is unknown. To determine the phenotype of DCs derived from the mature monocyte subset, the reverse transmigration model was used. In this system, monocytes develop into DCs while traversing through endothelial cells as well as the extracellular matrix, thus abolishing the need for addition of exogenous cytokines. ${ }^{17}$ To specifically study DCs originating from mature monocytes, this subset was isolated from PBMCs obtained from controls, labelled with carboxyfluorescein succinimidyl ester (CFSE) and remixed with the original PBMCs. This total population was then used in the reverse transmigration system. After $48 \mathrm{~h}$, the reverse transmigration fraction contained a clear population of DCs with cell surface ruffling and dendrites (fig $3 \mathrm{~A}$ ). This population contained CFSE+ and CFSE- cells, indicating that these cells are of CD16+ mature and CD16-immature origin. DCs derived from both monocyte subsets uniformly expressed HLA-DR at

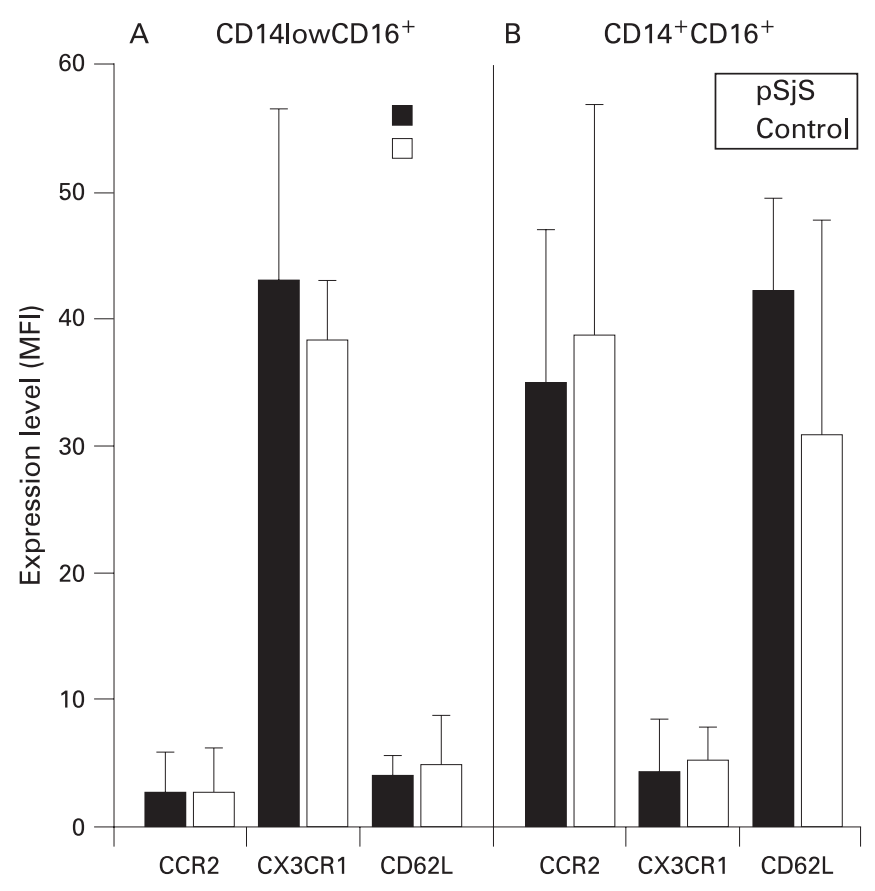

Figure 2 Normal chemokine receptor and adhesion molecule expression on primary Sjögren syndrome (pSjS) monocyte subsets. Peripheral blood mononuclear cells (PBMCs) were separated into CD16+ and CD16- populations using magnetic bead isolation and stained for CD14, chemokine (C-C motif) receptor 2 (CCR2), CD62L and CX3CR1. CD14 low $C D 16+(A)$ and CD14+CD16- (B) monocytes were gated and analysed by flow cytometry. Expression was calculated as mean fluorescence intensity (MFI) specific antibody-MFI isotype control, $\mathrm{n}=4-6$. 
Figure 3 Mature monocyte derived dendritic cells (DCs) partly express CD83 and DC-lysosome-associated membrane glycoprotein (LAMP). CD16+ monocytes were isolated from control peripheral blood mononuclear cells (PBMCs) by magnetic isolation and labelled using carboxyfluorescein succinimidyl ester (CFSE). Cells were then remixed and applied to the reverse transmigration system. A. After $48 \mathrm{~h}$, reverse transmigrated cells were harvested and showed ruffled membranes. B. DCs were harvested and CFSE+ and CFSE- DCs were analysed separately for marker expression by flow cytometry.

HLA-DR, human leukocyte antigen-DR; SIGN, specific intercellular adhesion molecule-3-grabbing non-integrin.
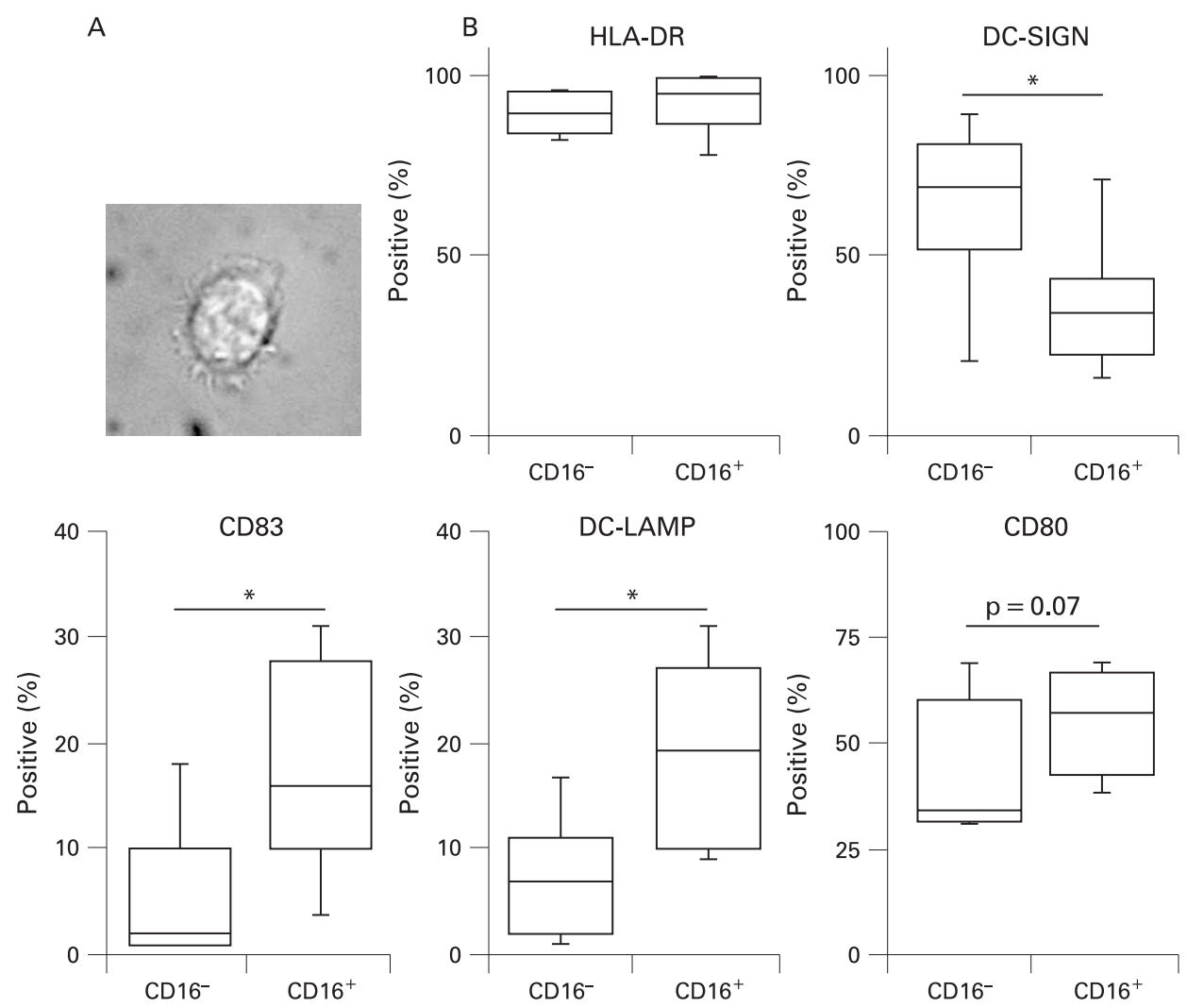

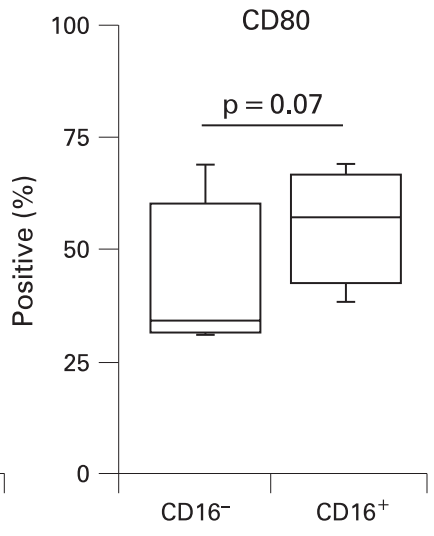

high levels (fig 3B). Previously, our group has reported the increased presence of $\mathrm{CD} 83+$ and $\mathrm{CD} 1 \mathrm{a}+\mathrm{DC}$ in the salivary glands of patients with pSjS. ${ }^{6}$ CD83 was present on a considerable proportion of DCs generated, especially in those generated from mature monocytes (fig 3B). CD1a+ DC were only rarely generated (2\%), and originated solely from immature monocytes (data not shown).

Recently, two more DC markers were described, DC-SIGN and DC-LAMP. ${ }^{29}{ }^{30}$ Expression of DC-SIGN was observed in up to $70 \%$ of DCs derived from mature monocytes, but was more common in DCs derived from the immature subset (up to $89 \%$, fig $3 \mathrm{~B}$ ). Contrary to this, expression of DC-LAMP was present in significantly larger proportions of DCs derived from mature monocytes (fig 3B). Similarly, expression of activation marker $\mathrm{CD} 80$ also tended to be higher in $\mathrm{CD} 14^{\text {low }} \mathrm{CD} 16+$ derived DCs (fig 3B).

\section{DC-LAMP+ DCs are present in lymphocytic infiltrates in pSjS salivary gland}

Data on the expression of DC-SIGN and DC-LAMP in the salivary glands of patients with $\mathrm{pSj}$ S has not been reported thus far. Therefore, expression of these markers was studied in labial or parotid biopsies obtained from four patients with $\mathrm{pSjS}$. Expression of DC-SIGN could readily be observed in all four patients in the lymphocytic infiltrates and scattered throughout the parenchyma (fig 4A). DC-LAMP+ cells were clearly present as well, with an expression pattern restricted to the lymphocytic infiltrates (fig 4A).

\section{Mouse homologue subset of mature monocytes migrates to salivary gland and preferentially develops into DCs}

To monitor monocyte trafficking in vivo, the NOD mouse model was used. This strain spontaneously develops lymphocytic infiltrates in the salivary glands similar to those seen in patients with $\mathrm{pSjS}^{7}$ and exhibits an expansion of Ly6C ${ }^{\text {low }}$ mature monocytes. ${ }^{23}$ Ly-6C ${ }^{\text {low }}$ mature and Ly- $6 C^{\text {high }}$ immature monocytes were labelled in vivo using latex beads labelled with the fluorescent marker Yellow-Green (YG). ${ }^{26}$ SMG were removed 4 days later and analysed for presence of YG+ cells by flow cytometry. Although SMG total cell suspensions show some autofluorescence, a clear population of YG+ cells could be observed in all mice (fig 5A), regardless of the monocyte subset labelled. The YG+ population in the SMG was then further analysed for expression of the dendritic cell marker CD11c. YG+ cells derived from mature monocytes cells were largely positive for CD11c (mean 59 (14)\%), indicating their dendritic nature (fig 5B). In contrast, expression of CD11c on YG+ cells derived from immature monocytes was significantly lower (mean 36 (8)\%, fig $5 B, C)$. This supports the idea that although both populations migrate to the SMG, mature monocytes preferentially develop into tissue DCs.

\section{DISCUSSION}

This study shows that in $\mathrm{pSjS}$ the frequency of CD16+ mature monocytes is increased compared to controls. Similar data has been described for patients with RA, ${ }^{13} 19$ although in our results, the increase in CD16+ monocytes in RA was less pronounced than that in $\mathrm{pSjS}$ (data not shown). The mature subset expresses normal levels of chemokine receptors and adhesion molecules. DCs derived from mature monocytes partly express DC-LAMP and CD83, a phenotype similar to DCs in $\mathrm{pSjS}$ salivary gland.

Increased levels of CD16+ monocytes have been described in systemic infection and sepsis, where cytokines were suggested to be the causal factor. ${ }^{21} 3132$ Since in our patient cohort no correlation between CD16+ frequency and these cytokines could be found, a straightforward cytokine effect appears to be 

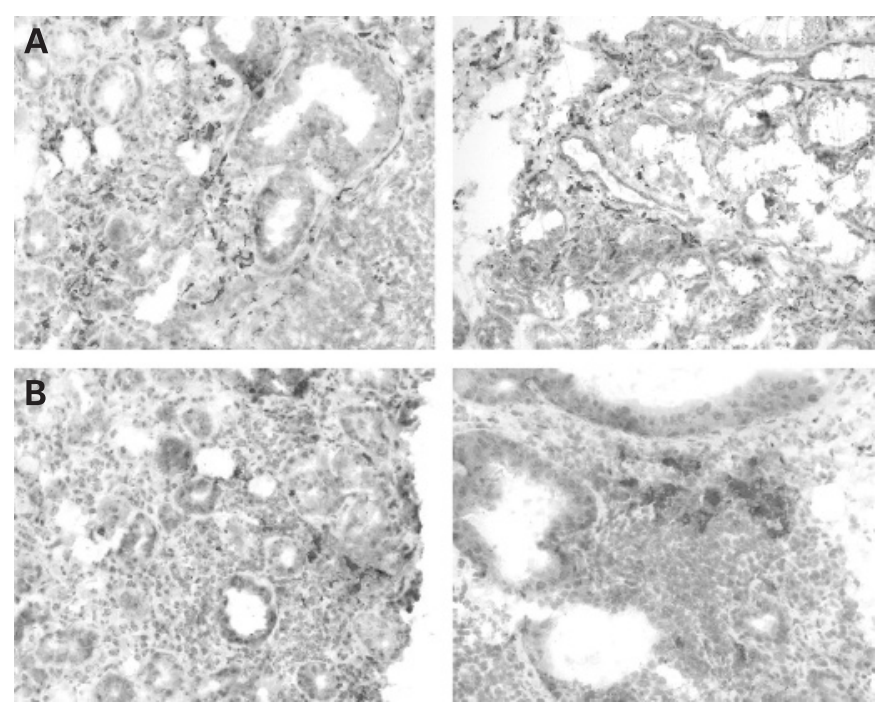

Figure 4 Dendritic cell (DC)-specific intercellular adhesion molecule-3grabbing non-integrin (SIGN) and DC-lysosome-associated membrane glycoprotein (LAMP) have different expression patterns in primary Sjögren syndrome (pSjS) salivary gland. Frozen parotid (left) or labial (right) biopsies of four pSjS patients were analysed by immunohistochemistry, representative stainings are shown. A. DCSIGN+ cells are scattered throughout the parenchyma (magnification $320 \times$ ). B. DC-LAMP+ cells are restricted to the focal infiltrates (magnification $320 \times$ ).

unlikely. Additionally, levels of mature monocytes did not correlate with disease duration, thus contradicting the idea that the increased frequency of this subset is simply due to an ongoing systemic inflammation.

The fate of mature monocytes after leaving the circulation has been poorly elucidated. Most data on the developmental fate of monocyte subsets has been derived from mouse studies that focused on the homologue population of Ly- $6 \mathrm{C}^{\text {low }}$ mature monocytes. ${ }^{33}$ A recent report has shown that Ly-6Clow monocytes can develop into lung DCs but not spleen DCs, ${ }^{34}$ thus indicating that this subset may be mainly involved in the seeding of DCs in non-lymphoid organs. In the NOD mouse, a spontaneous model for $\mathrm{pSjS},{ }^{22}$ an increase in mature monocytes and salivary gland infiltrating CD11c+ DCs was observed. ${ }^{73}$ This study shows that mature monocytes indeed have the capacity to migrate into the submandibular glands, and develop into CD11c+ cells more often then their immature counterparts. In mice, expression of CD11c is mainly found on DCs and alveolar macrophages, with only low levels being expressed by other macrophage subsets. ${ }^{35}{ }^{36}$ Given the high expression of

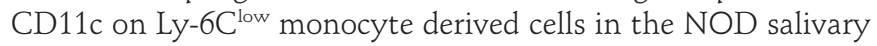
gland, these cells are most likely DCs. Recently, the NOD mouse model was reported to lack longitudinal stability with regard to the development of SjS-like symptoms. ${ }^{37}$ In the current study, the decrease in salivary flow was not analysed, so a direct link between monocyte infiltration and decreased salivary flow could not be established. However, the results do show that Ly$6 C^{\text {low }}$ monocytes are capable of migrating to the salivary glands and preferentially develop into DCs. Although the populations of CD16+ and Ly-6C low monocytes cannot be taken as completely identical, this strongly supports the idea that mature monocytes preferentially differentiate into tissue DCs.

The inhibitory FcGRIIb marker has been implicated in maturation of DCs from monocytes in humans. Blocking this receptor results in increased DC maturation and activation,
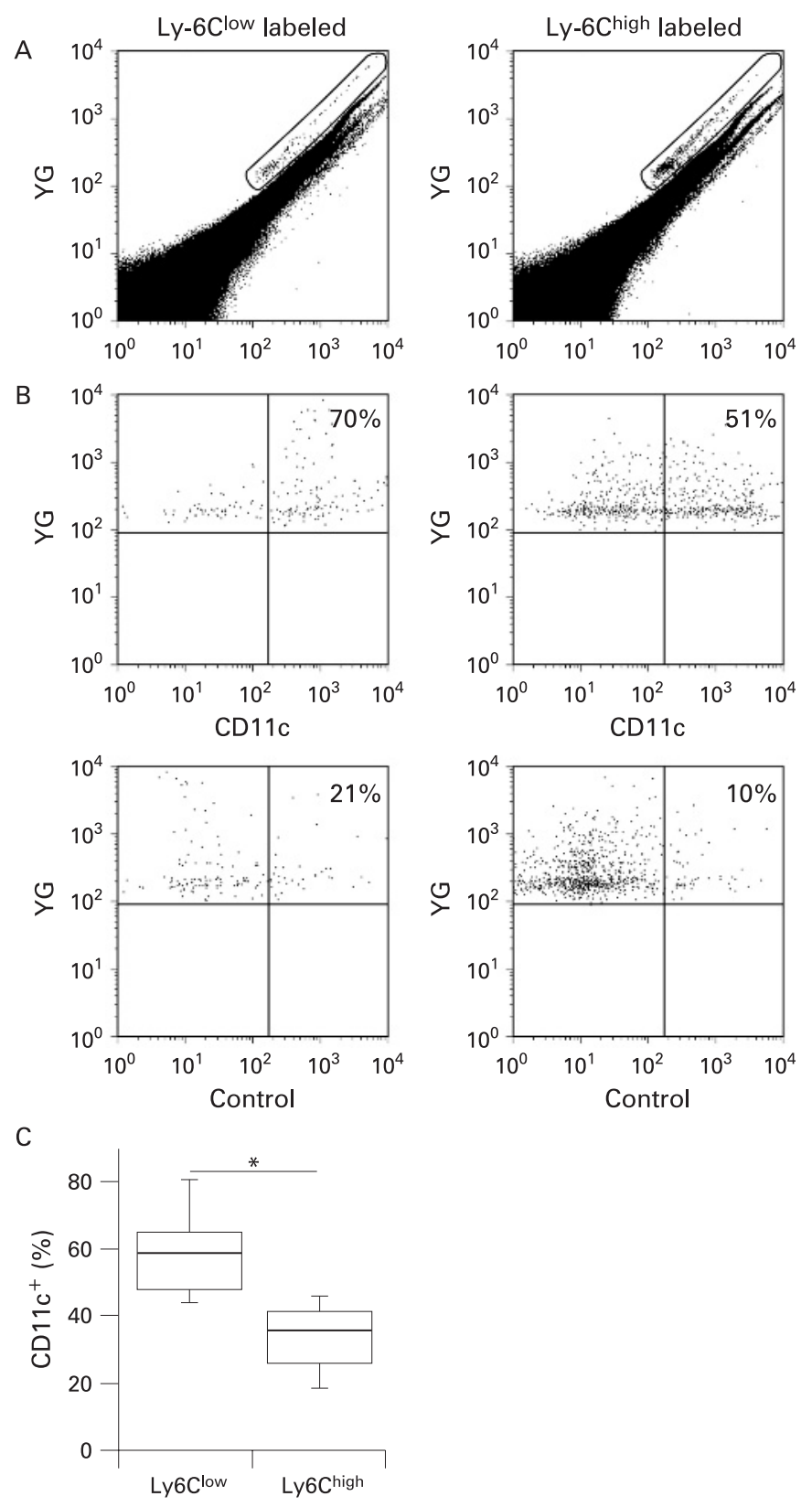

Figure 5 Mature mouse monocytes develop into salivary gland

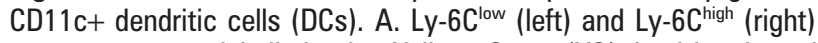
monocytes were labelled using Yellow-Green (YG)-dyed beads and presence of $\mathrm{YG}+$ cells in the submandibular glands (SMG) was analysed after 4 days. B. YG+ cells were gated and analysed for expression of CD11c, a representative picture of each labelling is shown. C. YG+ cells isolated from SMG were gated and analysed for expression of CD11c+, boxplots represent median with upper and lower quartiles, whiskers represent range $(n=7-10),{ }^{*} p<0.01$.

suggesting the FcGRIIb is a marker of relatively immature monocytes. ${ }^{38}$ Indeed, expression of this receptor is decreased in CD16+ monocytes compared to CD16- monocytes as determined at the mRNA level (data not shown), confirming the hypothesis that CD16+ monocytes are more prone to develop into DCs than their CD16- counterpart.

Previously our group has shown that increased numbers of CD83+ and CD1a+ DCs are present in labial salivary glands of patients with $\mathrm{pSjS} .{ }^{6}$ In addition to this, it is shown here that the recently described markers DC-SIGN and DC-LAMP29 30 are expressed by DCs in pSjS labial and parotid salivary glands, 
with expression of DC-LAMP restricted to the lymphocytic infiltrates. The importance of DCs for the formation of pathological infiltrates is shown by the fact that insulitis does not develop in the absence of macrophages and DCs. Furthermore, specific expansion of the DC population in a mouse model results in the formation of lymphocytic infiltration of the salivary glands. ${ }^{39}{ }^{40}$ To test if mature monocytes can develop in vitro into DCs displaying the phenotype seen in salivary glands, the reverse transmigration system was used. ${ }^{17}$ This system allows for monocytes to rapidly differentiate into DCs without the need for addition of cytokines. The cells used in this assay are obviously still capable of producing cytokines themselves, and these may impact the process in a paracrine fashion. Therefore, this system should not be considered as "cytokine-free", but rather as a closer resemblance of the physiological environment than cultures in the presence of excess amounts of exogenous cytokines. Both monocyte subsets developed into DCs expressing high levels of HLA-DR, with a large proportion of cells also expressing DC-SIGN. Expression of CD83 and DC-LAMP was detected in particular on DCs derived from mature monocytes. A similar trend was observed for CD80, indicative of a more activated DC phenotype. This is in line with previous observations, where increased expression of CD86 was observed in CD16+ derived DC. ${ }^{41}$ Only low numbers of CD1a+ DCs were detected, and originated solely from immature monocytes. This indicates that the CD1a+ DCs present in the SjS salivary gland may be a separate subset from the DCs displaying the activated phenotype. These results originate from in vitro experiments, which lack the local influence of the salivary gland. Cytokines produced by the glandular epithelium or infiltrating immune cells may also impact the differentiation of DCs, leading to different expression patterns than those described here. Therefore, although this data shows that mature monocytes have the capacity to develop into activated DCs, whether they preferentially do so specifically in salivary glands requires further confirmation in vivo.

In summary, patients with pSjS show increased numbers of mature CD16+ monocytes, which have the capacity to develop into CD83+ and DC-LAMP+ DCs, similar to the type of DC observed in salivary gland infiltrates in patients with $\mathrm{pSjS}$. Direct tracking of monocyte subsets is not possible in humans due to ethical restraints, but our studies in the NOD mouse support the model of the mature monocyte subset migrating to the salivary glands and preferentially developing into DCs.

Acknowledgements: The authors would like to thank GJ Randolph (Mount Sinai School of Medicine, New York, USA) for advice on the reverse transmigration and in vivo monocyte labelling models.

Competing interests: None declared.

Ethics approval: All subjects gave informed consent and experimental procedures were approved by the Medical Ethical Committees of Erasmus MC and University Medical Center Groningen.

\section{REFERENCES}

1. Larche MJ. A short review of the pathogenesis of Sjogren's syndrome. Autoimmun Rev 2006;5:132-5.

2. Kawakami A, Nakashima K, Tamai M, Nakamura H, Iwanaga N, Fujikawa K, et al. Toll-like receptor in salivary glands from patients with Sjogren's syndrome: functional analysis by human salivary gland cell line. J Rheumatol 2007;34:1019-26.

3. Spachidou MP, Bourazopoulou E, Maratheftis Cl, Kapsogeorgou EK, Moutsopoulos $H M$, Tzioufas AG, et al. Expression of functional Toll-like receptors by salivary gland epithelial cells: increased mRNA expression in cells derived from patients with primary Sjogren's syndrome. Clin Exp Immunol 2007;147:497-503.

4. Gottenberg JE, Cagnard N, Lucchesi C, Letourneur F, Mistou S, Lazure T, et al. Activation of IFN pathways and plasmacytoid dendritic cell recruitment in target organs of primary Sjogren's syndrome. Proc Natl Acad Sci USA 2006;103:2770-5.
5. Nordmark G, Alm GV, Ronnblom L. Mechanisms of disease: primary Sjogren's syndrome and the type I interferon system. Nat Clin Pract Rheumatol 2006;2:262-9.

6. van Blokland SC, Wierenga-Wolf AF, van Helden-Meeuwsen CG, Drexhage HA, Hooijkaas $\mathrm{H}$, van de Merwe JP, et al. Professional antigen presenting cells in minor salivary glands in Sjogren's syndrome: potential contribution to the histopathological diagnosis? Lab Invest 2000;80:1935-41.

7. van Blokland SC, van Helden-Meeuwsen CG, Wierenga-Wolf AF, Drexhage HA, Hooijkaas H, van de Merwe JP, et al. Two different types of sialoadenitis in the NODand MRL/lpr mouse models for Sjogren's syndrome: a differential role for dendritic cells in the initiation of sialoadenitis? Lab Invest 2000;80:575-85.

8. Shortman K, Liu YJ. Mouse and human dendritic cell subtypes. Nat Rev Immunol 2002;2:151-61.

9. Dubois B, Bridon JM, Fayette J, Barthelemy C, Banchereau J, Caux C, et al. Dendritic cells directly modulate B cell growth and differentiation. J Leukoc Biol 1999;66:224-30.

10. Balazs M, Martin F, Zhou T, Kearney J. Blood dendritic cells interact with splenic marginal zone B cells to initiate T-independent immune responses. Immunity 2002;17:341-52.

11. Hepburn AL, Mason JC, Davies KA. Expression of Fc $\gamma$ and complement receptors on peripheral blood monocytes in systemic lupus erythematosus and rheumatoid arthritis. Rheumatology (Oxford) 2004;43:547-54.

12. Bouma G, Coppens JM, Lam-Tse WK, Luini W, Sintnicolaas K, Levering WH, et al. An increased MRP8/14 expression and adhesion, but a decreased migration towards proinflammatory chemokines of type 1 diabetes monocytes. Clin Exp Immunol 2005;141:509-17.

13. Kawanaka N, Yamamura M, Aita T, Morita Y, Okamoto A, Kawashima M, et al. CD14+,CD16+ blood monocytes and joint inflammation in rheumatoid arthritis. Arthritis Rheum 2002;46:2578-86.

14. Ziegler-Heitbrock HW, Fingerle G, Strobel M, Schraut W, Stelter F, Schutt C, et al. The novel subset of CD14+/CD16+ blood monocytes exhibits features of tissue macrophages. Eur J Immunol 1993;23:2053-8.

15. Ziegler-Heitbrock L. The CD14+CD16+ blood monocytes: their role in infection and inflammation. J Leukoc Biol 2007;81:584-92.

16. Ancuta P, Rao R, Moses A, Mehle A, Shaw SK, Luscinskas FW, et al. Fractalkine preferentially mediates arrest and migration of CD16+ monocytes. J Exp Med 2003;197:1701-7.

17. Randolph GJ, Sanchez-Schmitz G, Liebman RM, Schakel K. The CD16(+) $(\mathrm{Fc} \gamma \mathrm{RIII}(+))$ subset of human monocytes preferentially becomes migratory dendritic cells in a model tissue setting. J Exp Med 2002;196:517-27.

18. Sunderkotter C, Nikolic T, Dillon MJ, Van Rooijen N, Stehling M, Drevets DA, et al. Subpopulations of mouse blood monocytes differ in maturation stage and inflammatory response. J Immunol 2004;172:4410-7.

19. Cairns AP, Crockard AD, Bell AL. The CD14+ CD16+ monocyte subset in rheumatoid arthritis and systemic lupus erythematosus. Rheumatol Int 2002;21:189-92.

20. Thieblemont N, Weiss L, Sadeghi HM, Estcourt C, Haeffner-Cavaillon N. CD14lowCD16high: a cytokine-producing monocyte subset which expands during human immunodeficiency virus infection. Eur J Immunol 1995;25:3418-24.

21. Fingerle G, Pforte A, Passlick B, Blumenstein M, Strobel M, Ziegler-Heitbrock HW The novel subset of CD14+/CD16+ blood monocytes is expanded in sepsis patients. Blood 1993:82:3170-6.

22. van Blokland SC, Versnel MA. Pathogenesis of Sjogren's syndrome: characteristics of different mouse models for autoimmune exocrinopathy. Clin Immunol 2002;103:111-24.

23. Nikolic T, Bouma G, Drexhage HA, Leenen PJ. Diabetes-prone NOD mice show an expanded subpopulation of mature circulating monocytes, which preferentially develop into macrophage-like cells in vitro. J Leukoc Biol 2005;78:70-9.

24. Vitali C, Bombardieri S, Jonsson R, Moutsopoulos HM, Alexander EL, Carsons SE, et al. Classification criteria for Sjogren's syndrome: a revised version of the European criteria proposed by the American-European Consensus Group. Ann Rheum Dis 2002;61:554-8.

25. Pijpe J, Kalk WW, van der Wal JE, Vissink A, Kluin PM, Roodenburg JL, et al. Parotid gland biopsy compared with labial biopsy in the diagnosis of patients with primary Sjogren's syndrome. Rheumatology (Oxford) 2007;46:335-41.

26. Tacke F, Ginhoux F, Jakubzick C, van Rooijen N, Merad M, Randolph GJ. Immature monocytes acquire antigens from other cells in the bone marrow and present them to T cells after maturing in the periphery. J Exp Med 2006;203:583-97.

27. Van Rooijen N, Sanders A. Liposome mediated depletion of macrophages: mechanism of action, preparation of liposomes and applications. J Immunol Methods 1994:174:83-93.

28. Iwahashi M, Yamamura M, Aita T, Okamoto A, Ueno A, Ogawa N, et al. Expression of Toll-like receptor 2 on CD16+ blood monocytes and synovial tissue macrophages in rheumatoid arthritis. Arthritis Rheum 2004;50:1457-67.

29. Geijtenbeek TB, Torensma R, van Vliet SJ, van Duijnhoven GC, Adema GJ, van Kooyk Y, et al. Identification of DC-SIGN, a novel dendritic cell-specific ICAM-3 receptor that supports primary immune responses. Cell 2000;100:575-85.

30. de Saint-Vis B, Vincent J, Vandenabeele S, Vanbervliet B, Pin JJ, Ait-Yahia S, et al A novel lysosome-associated membrane glycoprotein, DC-LAMP, induced upon DC maturation, is transiently expressed in MHC class II compartment. Immunity 1998; 9:325-36

31. Blumenstein M, Boekstegers $P$, Fraunberger $P$, Andreesen R, Ziegler-Heitbrock HW, Fingerle-Rowson G. Cytokine production precedes the expansion of CD14+CD16+ monocytes in human sepsis: a case report of a patient with self-induced septicemia. Shock 1997;8:73-5. 
32. Fingerle-Rowson G, Angstwurm M, Andreesen R, Ziegler-Heitbrock HW. Selective depletion of CD14+ CD16+ monocytes by glucocorticoid therapy. Clin Exp Immunol 1998;112:501-6

33. Geissmann F, Jung S, Littman DR. Blood monocytes consist of two principal subsets with distinct migratory properties. Immunity 2003;19:71-82.

34. Varol C, Landsman L, Fogg DK, Greenshtein L, Gildor B, Margalit R, et al. Monocytes give rise to mucosal, but not splenic, conventional dendritic cells. J Exp Med 2007;204:171-80.

35. Kruger T, Benke D, Eitner F, Lang A, Wirtz M, Hamilton-Williams EE, et al. Identification and functional characterization of dendritic cells in the healthy murine kidney and in experimental glomerulonephritis. J Am Soc Nephrol 2004;15:613-21.

36. Vermaelen K, Pauwels R. Accurate and simple discrimination of mouse pulmonary dendritic cell and macrophage populations by flow cytometry: methodology and new insights. Cytometry A 2004;61:170-77.

37. Lodde BM, Mineshiba F, Kok MR, Wang J, Zheng C, Schmidt M, et al. NOD mouse model for Sjogren's syndrome: lack of longitudinal stability. Oral Dis 2006;12:566-72.
38. Dhodapkar KM, Kaufman JL, Ehlers M, Banerjee DK, Bonvini E, Koenig S, et al. Selective blockade of inhibitory $\mathrm{Fc} \gamma$ receptor enables human dendritic cell maturation with IL-12p70 production and immunity to antibody-coated tumor cells. Proc Natl Acad Sci USA 2005;102:2910-5.

39. Nikolic T, Geutskens SB, van Rooijen N, Drexhage HA, Leenen PJ. Dendritic cells and macrophages are essential for the retention of lymphocytes in (peri)-insulitis of the nonobese diabetic mouse: a phagocyte depletion study. Lab Invest 2005;85:487-501.

40. Fujikado N, Saijo S, Yonezawa T, Shimamori K, Ishii A, Sugai S, et al. Dcir deficiency causes development of autoimmune diseases in mice due to excess expansion of dendritic cells. Nat Med 2008;14:176-80.

41. Sanchez-Torres C, Garcia-Romo GS, Cornejo-Cortes MA, Rivas-Carvalho A, Sanchez-Schmitz G. CD16+ and CD16- human blood monocyte subsets differentiate in vitro to dendritic cells with different abilities to stimulate CD4+ T cells. Int Immunol 2001;13:1571-81. 


\section{ARD}

\section{Increased frequency of CD16+ monocytes and the presence of activated dendritic cells in salivary glands in primary Sjögren syndrome}

M E Wildenberg, J M C Welzen-Coppens, C G van Helden-Meeuwsen, et al.

Ann Rheum Dis 2009 68: 420-426 originally published online April 8, 2008

doi: 10.1136/ard.2008.087874

Updated information and services can be found at:

http://ard.bmj.com/content/68/3/420.full.html

These include:

$\begin{array}{cl}\text { Data Supplement } & \begin{array}{c}\text { "web only appendix" } \\ \text { http://ard.bmj.com/content/suppl/2009/01/29/68.3.420.DC1.html }\end{array} \\ \text { References } & \begin{array}{c}\text { This article cites } 41 \text { articles, } 14 \text { of which can be accessed free at: } \\ \text { http://ard.bmj.com/content/68/3/420.full.html\#ref-list-1 }\end{array} \\ \begin{array}{c}\text { Email alerting } \\ \text { service }\end{array} & \begin{array}{l}\text { Receive free email alerts when new articles cite this article. Sign up } \\ \text { the box at the top right corner of the online article. }\end{array} \\ \begin{array}{c}\text { Topic } \\ \text { Collections }\end{array} & \begin{array}{c}\text { Articles on similar topics can be found in the following collections } \\ \text { Immunology (including allergy) (45820 articles) } \\ \text { Inflammation (13893 articles) }\end{array}\end{array}$

Notes

To request permissions go to:

http://group.bmj.com/group/rights-licensing/permissions

To order reprints go to:

http://journals.bmj.com/cgi/reprintform

To subscribe to BMJ go to:

http://group.bmj.com/subscribe/ 